University of Wollongong

Research Online

Faculty of Engineering and Information

Faculty of Engineering and Information

Sciences - Papers: Part A

Sciences

$1-1-2016$

\title{
A Comparative study of fluid flow and mass transfer in a trumpet-shaped ladle shroud using large eddy simulation
}

Jiangshan Zhang

University of Wollongong, jz822@uowmail.edu.au

Jingshe Li

University of Science And Technology Beijing

Yi Yan

University of Wollongong, yy619@uowmail.edu.au

Zhixin Chen

University of Wollongong, zchen@uow.edu.au

Shufeng Yang

University of Science And Technology Beijing

See next page for additional authors

Follow this and additional works at: https://ro.uow.edu.au/eispapers

Part of the Engineering Commons, and the Science and Technology Studies Commons

Research Online is the open access institutional repository for the University of Wollongong. For further information contact the UOW Library: research-pubs@uow.edu.au 


\title{
A Comparative study of fluid flow and mass transfer in a trumpet-shaped ladle shroud using large eddy simulation
}

\author{
Abstract \\ The advantages of trumpet-shaped ladle shrouds (TLS) have been frequently demonstrated over \\ conventional straight-bore ladle shrouds (CLS) with respect to production efficiency and molten steel \\ quality in continuous casting practices. The present study is to shed some lights on why the TLS are \\ better than the CLS design by examining the fluid dynamics and mass transfer using large eddy \\ simulation. The obtained numerical results were validated with particle imaging velocimetry experiments. \\ Flow velocity, deformation, turbulent energy dissipation, and mixing kinetics of tracer were discussed. The \\ results showed that the entering jet of the CLS flowed straight down into the tundish with a relatively high \\ speed (average at 0.710 to $0.815 \mathrm{~m} / \mathrm{s}$ ) and turbulent kinetic energy. However, the trumpet section of a \\ TLS intensified velocity differences, strain rates, and vortices, and promoted an increase on turbulence \\ dissipation rate in the interior of the ladle shroud. The average speed of the entering jet to the tundish was \\ decreased to 0.270 to $0.410 \mathrm{~m} / \mathrm{s}$ from the $0.708 \mathrm{~m} / \mathrm{s}$ of the inlet speed. The entering jet from the TLS \\ swung, twisted and well mixed with surrounding fluid in the tundish, and dissipated its kinetic energy. \\ Consequently, the turbulence of the whole flow field as well as the mean skin friction coefficient of \\ tundish wall and the velocity of free liquid surface were reduced. A tracer experiment was carried out to \\ study mass transfer and flow mixing behavior, and the results demonstrated that the use of the TLS \\ increased the plug volume and decreased the dead zone, thereby enhancing inclusion flotation.

\section{Disciplines} \\ Engineering | Science and Technology Studies

\section{Publication Details} \\ Zhang, J., Li, J., Yan, Y., Chen, Z., Yang, S., Zhao, J. \& Jiang, Z. (2016). A Comparative study of fluid flow \\ and mass transfer in a trumpet-shaped ladle shroud using large eddy simulation. Metallurgical and \\ Materials Transactions B: Process Metallurgy and Materials Processing Science, 47 (1), 495-507.

\section{Authors} \\ Jiangshan Zhang, Jingshe Li, Yi Yan, Zhixin Chen, Shufeng Yang, Jingwei Zhao, and Zhengyi Jiang
}




\title{
A Comparative Study of Fluid Flow and Mass Transfer in a Trumpet-Shaped
}

\section{Ladle Shroud Using Large Eddy Simulation}

\author{
Jiangshan Zhang, Jingshe Li, Yi Yan, Zhixin Chen, Shufeng Yang, \\ Jingwei Zhao, Zhengyi Jiang
}

Jiangshan Zhang, Ph.D. Student, is with the School of Metallurgical and Ecological Engineering, University of Science and Technology Beijing, Beijing, 100083, P.R. China, also with the School of Mechanical, Materials and Mechatronic Engineering, University of Wollongong. Jingshe Li, Professor and Shufeng Yang, Associate Professor, are with the School of Metallurgical and Ecological Engineering, University of Science and Technology Beijing. Zhengyi Jiang, Professor, Zhixin Chen, Senior Lecturer, Yi Yan, Ph.D. Student and Jingwei Zhao, Research Fellow, are with the School of Mechanical, Materials and Mechatronic Engineering, University of Wollongong.

Corresponding authors:

Zhengyi Jiang, jiang@uow.edu.au, School of Mechanical, Materials and Mechatronic Engineering, University of Wollongong, Wollongong, NSW 2522, Australia. Tel.: +61 24221 4545; fax: +61 2 42215474

Shufeng Yang, yangshufeng@ustb.edu.cn, School of Metallurgical and Ecological Engineering, University of Science and Technology Beijing, Beijing, 100083, P.R. China. Tel.:+8618511695668 
ABSTRACT: The advantages of trumpet-shaped ladle shrouds (TLS) have been frequently demonstrated over conventional straight-pore ladle shrouds (CLS) with respect to production efficiency and molten steel quality in continuous casting practices. The present study is to shed some lights on why the TLS are better than the CLS design by examining the fluid dynamics and mass transfer using Large Eddy Simulation (LES). The obtained numerical results were validated with Particle Imaging Velocimetry (PIV) experiments. Flow velocity, deformation, turbulent energy dissipation and mixing kinetics of tracer were discussed. The results showed that the entering jet of the CLS flowed straight down into the tundish with a relatively high speed (average at $0.710 \sim 0.815 \mathrm{~m} / \mathrm{s}$ ) and turbulent kinetic energy. However, the trumpet section of a TLS brought about velocity differences, strain rates and vortices, and promoted an increase on turbulence dissipation rate in the interior of the ladle shroud. The average speed of the entering jet to the tundish was decreased to $0.270 \sim 0.410 \mathrm{~m} / \mathrm{s}$ from the $0.708 \mathrm{~m} / \mathrm{s}$ of the inlet speed. The entering jet from the TLS swung, twisted and well mixed with surrounding fluid in the tundish and dissipated its kinetic energy. Consequently, the turbulence of the whole flow field as well as the mean skin friction coefficient of tundish wall and the velocity of free liquid surface were reduced. A tracer experiment was carried out to study mass transfer and flow mixing behaviours, and the results demonstrated the use of the TLS increased the plug volume and decreased the dead zone, thereby enhancing inclusion flotation.

KEYWORDS: large eddy simulation, trumpet-shaped ladle shroud, conventional ladle shroud, mass transfer, flow structure. 


\section{Introduction}

A tundish is basically regarded as an intermediate reservoir to receive molten steel from a ladle and deliver it into a mold (s) in continuous casting. Metallurgists have been trying to optimize the tundish operation and make the best use of this vessel to explore as many as functions as possible, such as inclusion removing, alloying and temperature adjustment. Fluid flow is of great significance to determine the tundish performance and has drawn particular attentions in the past decades. ${ }^{[1]}$. A variety of flow control devices (FCDs), for instance, weir, dam and turbulent inhibitor ${ }^{[2-5]}$, have received a lot of past studies and been applied to control the fluid dynamics in steelmaking process. It is a new concept to employ and design a ladle shroud as a FCD to improve the flow pattern of a tundish ${ }^{[6]}$. A conventional ladle shroud (CLS) is typically a straight bore nozzle made of refractory materials, serving as a flow channel between a ladle and a tundish to protect the liquid steel. While, the CLS was reported to be suffering several defects ${ }^{[7-9]}$, namely air pick-up, nozzle clogging, slag-eye around the ladle shroud etc., which need to be minimized or avoided. Apart from the newly-emerged swirling ladle shroud (SLS) ${ }^{[10]}$, dissipative ladle shroud $(\mathrm{DLS})^{[6,11]}$ and inert gas injection ${ }^{[12]}$, trumpet-shaped ladle shroud (TLS also known as bell-shaped ladle shroud ${ }^{[8,13,14]}$ is one of the designs that are widely applied in steelmaking plants. The TLS is characterized with a trumpet tip with gradually enlarged diameter which changes the flow patterns both inside the ladle shroud and the tundish. Schematic drawings of the TLS and the CLS are shown in Figure 1(a). The documented advantages of the TLS over the CLS are summarized in Table I. This trumpet-shaped ladle shroud was mainly suggested for its merits in two aspects: production efficiency and molten steel quality. The TLS was demonstrated to be able to decrease 
the rate of nozzle clogging ${ }^{[8,13,15]}$, accommodate for hot gas pockets, and eliminate flow backs during a submerged ladle change or first tundish filling operation ${ }^{[15,16]}$. These effectively reduce the frequency of operation disruptions and enhance the production efficiency. Meanwhile, the TLS is capable of improving flow characteristics in a tundish ${ }^{[14]}$, minimizing molten steel turbulence ${ }^{[8]}$ and eliminating slag entrapment ${ }^{[7,13]}$, thereby improving the steel cleanness with lower total oxygen (T.O) content and defects ${ }^{[9]}$. With the help of the TLS, a decreased folding rate was reported during cold rolling in Inland Steel Company ${ }^{[13]}$. Although the above mentioned benefits are frequently confirmed for this commercialized ladle shroud, the mechanism(s), related to fluid flow and mass transfer in TLS and tundish, has not been sufficiently investigated mainly due to the difficulty to directly monitor the molten steel in a continuous casting process and the limitation of experimental methods.

With the development of computing science and computer technology, numerical simulation gets rapidly developed and facilitates the research in Metallurgical Engineering, especially in computational fluid dynamics (CFD). Mathematical modelling generally falls into the categories of Reynolds-averaged approach, Large Eddy Simulation (LES) and Direct Numerical Simulation (DNS $)^{[17]}$. The Reynolds-averaged approach, typically with the two-equation $(k-\varepsilon)$ turbulence model, has been extensively adopted thanks to its low time consumption and requirement to hardware. However, it fails to provide reliable results for swirling flows and highly strained angular velocities of rotating flows ${ }^{[18]}$. The DNS solves the Navier-Stokes (N-S) equations directly but at a high computational cost. The LES is a method sits between the two-equation turbulence model and DNS. In the LES the N-S equations are firstly filtered; the filtered equations are then simulated and the eddies with sizes smaller than that the filter size are modeled ${ }^{[19,20]}$ to get 
relatively accurate data at an acceptable short computing time.

The present work aims at investigating the flow structure and mass transfer in a trumpet-shaped ladle shroud (TLS) and a tundish model using the LES, compared with that in a conventional ladle shroud (CLS). The flow velocity, deformation, and turbulent energy dissipation are discussed to analyze the flow field and the mechanisms of reported benefits of the TLS.

\section{Mathematical modelling}

\section{A. Geometrical Description}

Models with a size of one-third scale of the real CLS and the TLS were used for the physical and mathematical simulations. A schematic view of the two ladle shrouds is shown in Figure 1 (a) and their dimensions are listed in Table II. The diameters of the inlet and the outlet are 0.030 and $0.050 \mathrm{~m}$ respectively for the TLS. One of the main purposes of the current study is to investigate the flu dynamics inside the ladle shroud which mainly influences the surrounding fluid of the jet. The inside flow structures of the two ladle shrouds are not significantly affected by the geometry of a much larger tundish. The structure of the tundish was simplified as a rectangular tank so that high quality and fine grid blocks can be fitted for accurate simulations. The computational domain was meshed into $\sim 1,750,000$ hexahedral cells (see Figure 1(b)) with the finest spacing of 0.215 $\mathrm{mm}$ near the wall of the shroud in XY plane. The maximum mesh size is less than $4 \mathrm{~mm}$ inside the tundish. Water was used as the fluid in all the simulations.

\section{B. Mathematical Formulation and Procedure}

In the LES model, only large scale structures are directly resolved, while small eddies are modeled. Compared with large scales, the smallest scales are more homogeneous and less affected 
by the boundary conditions, hopefully making the modelling of the small scales to be simpler. A Subgrid-scale (SGS) model, Wall-Adapting Local Eddy-Viscosity (WALE) model proposed by Nicoud and Ducros ${ }^{[21]}$, is employed to represent the effects of the unresolved small-scale fluid motions in the equations that are governing the large-scale motions in computer modelling.

In the SGS model, each quantity $\phi$ is decomposed as $\phi=\bar{\phi}+\phi^{\prime \prime} . \bar{\phi}$ is the resolvable-scale component and $\phi^{\prime \prime}$ is the sub-grid (unresolved) component. After a filtering procedure to the local and instantaneous quantities, the $\bar{\phi}$ is obtained. In FLUENT, the finite-volume discretization implicitly offers the filtering operation as ${ }^{[22]}$ (filtered variables are denoted with an overbar):

$$
\bar{\phi}(x)=\frac{1}{V} \int_{V} \phi\left(x^{\prime}\right) G\left(x, x^{\prime}\right) d x^{\prime}, x^{\prime} \in V
$$

where $V$ is the volume of the computational cell. The filter function $G\left(x, x^{\prime}\right)$ determines the size and structure of the small scales and is implied as:

$$
G\left(x, x^{\prime}\right)\left\{\begin{array}{l}
1 / V, \quad x^{\prime} \in V \\
0, x^{\prime} \text { otherwise }
\end{array}\right.
$$

The filtered continuity and Navier-Stokes equations for incompressible fluids are as follows:

$$
\begin{gathered}
\frac{\partial \bar{u}_{i}}{\partial x_{i}}=0 \\
\frac{\partial \bar{u}_{i}}{\partial t}+\frac{\partial\left(\bar{u}_{i} \bar{u}_{j}\right)}{\partial x_{j}}=-\frac{1}{\rho} \frac{\partial \bar{p}}{\partial x_{i}}+\frac{\partial}{\partial x_{j}}\left(v_{\text {eff }}\right)\left(\frac{\partial \bar{u}_{i}}{\partial x_{j}}+\frac{\partial \bar{u}_{j}}{\partial x_{i}}\right)-\frac{\partial \tau_{i j}}{\partial x_{j}} \\
v_{\text {eff }}=v_{0}+v_{\mathrm{t}}
\end{gathered}
$$

where, $\rho$ is density. $t$ is time. $p$ and $u_{i}$ are the pressure and filtered velocities respectively. $v_{0}$ and $v_{t}$ represent the molecule viscosity and eddy-viscosity, which are summed together as effective viscosity $v_{\text {eff }}$. The subscripts $i$ and $j$ are the three directions in Cartesian coordinates. $\tau_{i j}$ represents the unknown SGS stresses and defined by:

$$
\tau_{i j}=\overline{u_{i} u_{j}}-\bar{u}_{i} \bar{u}_{j}
$$


After filtering operation, the SGS stresses are still unknown and require proper modelling. An eddy-viscosity $\left(v_{\mathrm{t}}\right)$ assumption (Boussinersq's hypothesis) is made in the FLUENT package to model the stress tensor as follows:

$$
\tau_{i j}=\frac{1}{3} \vartheta_{i j} \tau_{k k}+2 v_{\mathrm{t}} \bar{S}_{i j}
$$

where $\vartheta_{i j}$ is the stress tensor due to molecular viscosity; the isotropic part of the SGS stresses $\tau_{k k}$ is added to the filtered static pressure term or neglected for incompressible flows. $\bar{S}_{i j}$ is the rate of strain tensor defined by:

$$
\bar{S}_{i j}=\frac{1}{2}\left(\frac{\partial \bar{u}_{i}}{\partial x_{j}}+\frac{\partial \bar{u}_{j}}{\partial x_{i}}\right)
$$

Turbulent viscosity also needs to be modelled to close the equations. Among several popular SGS models, including Smagorinsky-Lilly model, the WALE model and dynamic kinetic energy SGS model ${ }^{[22]}$, the WALE model is based on a tensor invariant $\left(v_{\mathrm{t}}=O\left(y^{3}\right)\right)$ and reproduces the proper scaling at the wall, which is believed to be reasonable and accurate in flows associated with complicate geometries ${ }^{[21]}$. To be more specific, this model captures the expected variation of eddy viscosity with the cube of distance close to the wall, avoiding the difficulty of any expensive or complicated dynamic procedure or need of Van-driest damping as a function of $y+$ in a complex geometry ${ }^{[21]}$. The WALE model is sensitive to both the strain and the rotation rate of small turbulent structures and is suitable for the flow structure inside the shroud and tundish. The $v_{\mathrm{t}}$ is given as ${ }^{[22]}$ :

$$
v_{\mathrm{t}}=\rho L_{s}^{2} \frac{\left(S_{i j}^{d} S_{i j}^{d}\right)^{3 / 2}}{\left(\bar{S}_{i j} \bar{S}_{i j}\right)^{5 / 2}+\left(S_{i j}^{d} S_{i j}^{d}\right)^{5 / 4}}
$$

where $L_{s}$ is the mixing length for sub-grid scales, defined as below:

$$
L_{s}=\min \left(\kappa d, C_{w} V^{1 / 3}\right)
$$


where $d$ is the distance from the cell center to the closest wall; the von Kármán constant $\kappa$ is 0.418. $C_{w}$ is chosen to be 0.325 , which has been confirmed to yield satisfactory results for a wide range of flow in FLUENT $^{[22]}$. In LES, the eddy-viscosity should be unchangeable when the frame of the reference is changed. This requires invariants of a tensor to be representative of the turbulence to support an operator of the space and the time. The velocity gradient tensor $\bar{g}_{i j}=\partial \bar{u}_{i} / \partial x_{j}$ is believed to be a good candidate to represent the velocity fluctuations at the length scale. For WALE model, the operator is built by considering the traceless symmetric part of the square of the velocity gradient tensor $\left(\bar{g}_{i j}^{2}=\bar{g}_{i j} \bar{g}_{k j}\right)^{[21]}$ :

$$
S_{i j}^{d}=1 / 2\left(\bar{g}_{i j}^{2}+\bar{g}_{j i}^{2}\right)-1 / 3 \delta_{i j} \bar{g}_{k k}^{2}
$$

where the Kronecker symbol $\delta_{i j}=1$, if $i=j$, else $\delta_{i j}=0$.

The governing equations were discretized and calculated using finite volume method with $2^{\text {nd }}$-order central differencing scheme for convection terms, which was carried out in the FLUENT software package. Implicit Fractional Step Method (IFSM) was employed to realize the velocity-pressure coupling. The time integration was performed with $2^{\text {nd }}$ order implicit scheme. The time-dependent LES modeling started from a steady state obtained by the $k$ - $\varepsilon$ two-equation model in the whole domain. The flow was allowed to develop for 20 seconds before collecting time statistics with a time step $\Delta t=0.0005$ second. Time statistics were collected for 80 seconds to get average values.

For the tracer experiments, an instantaneous tracer transfer associated with fluid flow was modeled by solving the following equations:

$$
\frac{\partial C}{\partial t}+u \frac{\partial C}{\partial x}+v \frac{\partial C}{\partial y}+w \frac{\partial C}{\partial z}=D_{\text {eff }}\left(\frac{\partial^{2} C}{\partial x^{2}}+\frac{\partial^{2} C}{\partial y^{2}}+\frac{\partial^{2} C}{\partial z^{2}}\right)
$$

where $D_{\text {eff }}$ is the effective mass transfer coefficient, which is the summation of molecular and turbulent 
diffusivities:

$$
D_{e f f}=D_{0}+\frac{v_{\mathrm{t}}}{\rho \mathrm{Sc}_{\mathrm{t}}}
$$

where $D_{0}$ is the molecular diffusivity of a tracer, and $v_{\mathrm{t}}$ is the turbulent viscosity. As turbulent flow dominates the mass transfer, the Schmidt number $\mathrm{Sc}_{\mathrm{t}}$ was chosen to be one. The solutions of the species transfer equations were coupled with the solution of the LES model to acquire more reliable unsteady mass distribution. The theories of determining the volume fraction of the dead zone, plug volume and mixed volume have been well developed in single-strand tundish in reference ${ }^{[23]}$.

\section{Boundary Conditions}

1) Inlet: a constant inflow velocity $(0.708 \mathrm{~m} / \mathrm{s})$ profile was applied at the nozzle inlet;

2) Outlet: a fixed pressure of $0 \mathrm{~Pa}$ (relative to the ambient) was applied at the outlet of the tundish;

3) Top surface and walls: free-slip boundaries with zero normal velocity were used at the top surface. All walls of the domain were considered to be no-slip, and the wall boundary domain was handled using the Werner-Wengle formulation ${ }^{[24]}$.

\section{Physical Modelling and Model Validation}

A water model was built to validate the mathematical model (LES) with the same flow parameters (geometries and flow rate etc.). A schematic view of the physical experiment apparatus is shown in Figure 2. PIV was used to measure the outflow velocity of the TLS. When the flow pattern reached to a steady state in the water model, data were collected.

Figure 3 shows three typical pairs of the flow field under the outlet of the TLS obtained from the PIV and LES. The main jet flows straight down (Figures 3 (a), (b)), to the left side (Figures 3 (c), (d)) and the right side (Figures 3 (e), (f)) respectively, which show that the velocity vectors of the LES model matched well with those of the PIV as a whole. The main stream flows out from 
the outlet at around $0.30 \mathrm{~m} / \mathrm{s}$, less than a half of the inlet speed. As the outflow is mixed with the fluid in the tundish, the stream swings, gets distorted, and its speed deceases rapidly to nearly 0.1 $\mathrm{m} / \mathrm{s}$ at the bottom of the tundish. Several vortices can also be observed on the both sides of the outflow owing to velocity differences among fluid elements. The speed reaches as low as less than $0.05 \mathrm{~m} / \mathrm{s}$ in the vortex regions. However, the location and dimension of the vortices, are not sufficiently consistent between the flow vector profiles obtained from the LES and PIV experiments as shown in Figure 3. The vector directions are not well matched at the right side of the jet in Figure 3 (a), (b) and (c), (d). It is actually challenging to get two same vector profiles for the numerical and physical results, because the flow patterns are extremely diverse and time-dependent. It also should be noted that the time steps are $0.02 \mathrm{~s}$ in the PIV measurements and $0.0005 \mathrm{~s}$ in the LES modeling respectively, which makes the PIV results seem more stable and simple than that of numerical simulations. Due to the setting and limitation of the PIV monitoring, the node spaces are also relatively coarser for the vectors in the PIV results than those in the LES modelling. It is, however, sufficient to capture the major characteristics of the turbulence flow to verify the LES simulations.

\section{Results and discussion}

\section{A. Flow Structure Inside the Ladle Shrouds}

\section{Velocity Distribution}

Velocity is the most directly perceived index to reflect the flow structure. Velocity fields of the two ladle shrouds were monitored at certain points, central lines and transversal planes (XZ plane) to display the turbulent flows respectively. 
Figure 4 shows ten-second instantaneous velocity histories at three points (p1, p2 and p3, located at the center, $1 / 2$ and $1 / 4$ of the outlet radius respectively). In the CLS, the velocity magnitude at $\mathrm{p} 1$ and $\mathrm{p} 2$ ranges from $0.80 \mathrm{~m} / \mathrm{s}$ to $0.83 \mathrm{~m} / \mathrm{s}$, and averages at $0.815 \mathrm{~m} / \mathrm{s}$. The speed at p3 varies between $0.62 \mathrm{~m} / \mathrm{s}$ and $0.76 \mathrm{~m} / \mathrm{s}$ and the average speed at $\mathrm{p} 3$ is the lowest at $0.71 \mathrm{~m} / \mathrm{s}$. These mean that the speeds of the three points are slightly accelerated in the vertical straight bore, when the inlet velocity magnitude is $0.708 \mathrm{~m} / \mathrm{s}$. In the TLS, the speeds at the three points seem to be much lower than those of the CLS due to the enlarged inner diameter. The speed at $\mathrm{p} 3$ exhibits the strongest fluctuations (from $0.05 \mathrm{~m} / \mathrm{s}$ to $0.40 \mathrm{~m} / \mathrm{s}$ ) and the standard deviation is $0.063(0.028$ for the CLS). Meanwhile, the standard deviations at $\mathrm{p} 1$ and $\mathrm{p} 2$ are 0.038 and 0.061 respectively in the TLS which are more than ten times larger than those of the CLS (0.003 and 0.006 respectively). These indicate that the outflow velocity of the TLS gets spread with larger fluctuations rather than focusing at center with a higher speed and less fluctuation.

The turbulent flow is very complex to be described in detail and the flow velocity varies with time stochastically. Therefore, three typical velocity profiles (denoted as modes 1 to 3 at $88.0 \mathrm{~s}$, $88.2 \mathrm{~s}$ and $88.4 \mathrm{~s}$ ) are chosen to show the flow characteristics at the centerlines of the two ladle shrouds as shown in Figure 5. The velocity magnitude increases continuously throughout the CLS due to the gravity force. In the TLS(see Figure 5(b)), the speed starts at $0.708 \mathrm{~m} / \mathrm{s}$ at the inlet and gradually goes up in the straight section and then the velocity begins to decline in the trumpet section. At the last $100 \mathrm{~mm}$ part of the TLS, obvious fluctuations can be seen due to the divergent volume and velocity difference among the fluid elements. The velocity fluctuation or difference promotes the rate at which turbulence kinetic energy is converted into thermal energy $(\varepsilon)$. With the dissipative effect of the ladle shroud and the divergent outlet, the stream gets divided and the 
outlet speed decreases to nearly a half of the inlet speed with wide fluctuations. The turbulent energy, introduced into the tundish slag layer, is closely associated with the entering jet velocity. A reduced velocity should thereby reduce impact forces of the flow on the tundish bottom and intensive slag-steel emulsification.

Figure 6 shows two typical velocity vector profiles and their enlarged views at the central XZ plane for the CLS and the TLS. The velocity continually rises in the straight pore in the CLS. Smaller velocity vectors are observed along the wall boundaries and virtually no vortex can be visualized due to the limited mesh density and capability of the LES model to capture the small-scale vortex ${ }^{[25]}$. In Figure 6(b) for the TLS, the main stream expands and the speed decreases in the trumpet section. Several vortices with upward flow vectors are formed along the wall in areas (1) and (2). Concurrently, distorted vectors, in curved paths, are created in area (3).

Although the nozzle clogging basically occurs at the entrance to the inverted nozzle and the entrance and at the change of section ${ }^{[26]}$ (conical to cylindrical, which is not considered here), it is also extended to the lower part of a nozzle. The nozzle clogging is influenced by the outflow of the nozzle and the effect of the TLS to reduce nozzle clogging has been reported ${ }^{[13]}$ as well. It is commonly believed that lessening the transport of inclusions to the nozzle walls is an effective strategy to eliminate clogging. Firstly, the trumpet part of the ladle shroud enlarges the volume of the nozzle and limits the transport and attachment of inclusions to the inner wall of the ladle shroud. Secondly, the low velocity reduces the blockage tendency in larger diameter nozzle ${ }^{[8,26]}$. Thirdly, the upward and distorted flow strengthens the forces to remove the deposited solid inclusions (deemed as the most important reason of nozzle clogging ${ }^{[8]}$ ) from the wall. These three aspects are probably the benefits and mechanisms of the TLS to alleviate nozzle clogging. 


\section{Deformation and turbulent energy dissipation}

The deformation and energy dissipation are important features of a turbulent flow, which can be used to characterize the flow structure in the ladle shroud. Deformation tensor $\left(D_{i j}\right)$ illustrates how fluid elements deform as a result of fluid motion, which is expressed as:

$$
D_{i j}=\frac{\partial u_{i}}{\partial x_{j}}=\frac{1}{2}\left(\frac{\partial u_{i}}{\partial x_{j}}+\frac{\partial u_{j}}{\partial x_{i}}\right)+\frac{1}{2}\left(\frac{\partial u_{i}}{\partial x_{j}}-\frac{\partial u_{j}}{\partial x_{i}}\right)=S_{i j}+\varsigma_{i j}
$$

where $S_{i j}=\frac{1}{2}\left(\frac{\partial u_{i}}{\partial x_{j}}+\frac{\partial u_{j}}{\partial x_{i}}\right)$, and $S_{i j}=\frac{1}{2}\left(\frac{\partial u_{i}}{\partial x_{j}}-\frac{\partial u_{j}}{\partial x_{i}}\right) \cdot S_{i j}$ is the symmetric part of the deformation tensor, which represents the rate of shear strain tensor for incompressible fluid; $\varsigma_{i j}$ is the asymmetric part and the vorticity tensor. The relationship between the dissipation rate of kinetic energy and the strain rate fluctuation can be expressed as:

$$
\varepsilon=2 v_{\text {eff }} \overline{s_{i j} s_{i j}}
$$

It indicates that the mean square of strain rate fluctuations is proportional to the dissipation rate.

Strain rate fluctuations are able to reflect the intensity of the dissipation rate. Strain rate profiles (denoted as mode 1 to 3 at $81.0 \mathrm{~s}, 82.2 \mathrm{~s}$ and $83.0 \mathrm{~s}$ ) at the centerlines for the two ladle shrouds are given in Figure 7. The increased strain rate is mainly due to the velocity difference between neighbor fluid elements. In the CLS, the strain rate rises from 2 to $17 \mathrm{~s}^{-1}$ with small fluctuations because of the continuously increased velocity inside the nozzle. The velocity keeps increasing along the CLS, which strengthens the velocity difference between fluid elements in the center and elements near the wall (velocity is zero). In the TLS, the strain rate fluctuates and slightly increases in the straight section, and climbs especially in the trumpet end to nearly $60 \mathrm{~s}^{-1}$ owing to the sudden change of volume and flow pattern. Thus, the strain rate of the TLS is larger and fluctuates more apparently than that of the CLS, which encourages more vortices and larger 
turbulence dissipation rates and finally makes the flow at the outlet velocity fluctuate but smaller speeds.

Vorticity is a measure of the rotation of a fluid element as it travels in the flow field. It is defined as the curl of the velocity vector:

$$
\zeta=\nabla \times u_{i}
$$

The dissipation rate of kinetic energy is proportional to the mean square fluctuations of the vorticity vector, which can be illustrated in the following equation.

$$
\varepsilon=v \overline{\varsigma_{i} \varsigma_{i}}
$$

Therefore, the turbulence energy dissipation can be characterized with the vortex magnitude in the fluid. Figure 8 shows the vorticity field and the evolution tracks of typical vortices in the XZ plane with the two shrouds. The velocity of the CLS is relatively high in magnitude when the jet hits against the fluid inside the tundish, resulting in the most intense vortices surrounding the jet. Several vortices of the magnitude nearly $300 \mathrm{~s}^{-1}$ are generated. As the stream flows down into the tundish, the volumes of the vortices get enlarged and their magnitudes are reduced to $150 \mathrm{~s}^{-1}$ or less (as circled in Figure 8(a)). The vortices ultimately dissipate into many small vortices, form new vortices and gradually disappear inside the tundish, in the company with the dissipated turbulent kinetic energy. It should be noted that the entering jet of a ladle shroud, with strong vortices and high velocity, is detrimental and not desirable due to its tendency to bring about surface turbulence and lead into slag entrapment. In the TLS, however, the strongest vortices occur at the joint part of the straight and trumpet sections inside the shroud. They gradually dissipate into numerous small eddies along the wall of the TLS and converge in the tundish, interact and get further dissipated. The inlet velocities of the two ladle shrouds are the same, the 
vorticity magnitude for the CLS is about $350 \mathrm{~s}^{-1}$ but, the vorticity magnitude only maximizes at $190 \mathrm{~s}^{-1}$ in the TLS. Vorticities of even smaller magnitude can be observed in the whole tundish with the TLS as well. The documented braking effect on the fluid illustrates the same behavior with a bell-shaped tip of the ladle shroud ${ }^{[18]}$. Hence, with the help of the trumpet section in altering the flow patterns both inside the ladle shroud and the tundish, the TLS changes the turbulence of the complete flow field as well as the location of the strongest vortex and turbulence, thereby being potential to diminish the liquid level fluctuation and the tendency of entrapping tundish slag.

\section{B. Tundish flow analysis}

The fluid flow inside the tundish is both a validating index to test the effects of an existing ladle shroud and the reason of designing a new ladle shroud. For this, four typical instantaneous velocity contours (main stream flowing straight downward, towards the right, towards the left, and in twist profile) of the tundish with each ladle shroud are calculated and shown in the $\mathrm{XZ}$ plane (same as that plane shown in Figure 8) in Figures 9 and 10. The downstream mixes with the fluid in the tundish, flows straight down, impacts at the bottom wall where it is divided into substreams as shown in Figures 9(a) and 10(a). The jet speed from the CLS is much higher than that from the TLS. The downstream of the CLS straightly goes down to the tundish with an accelerated speed which can reach nearly $0.85 \mathrm{~m} / \mathrm{s}$ inside the tundish. The same situation happens in the other three pairs of contours, exerting strong impact pressures and forces on the bottom lining of the tundish. The outlet streams of the TLS get twisted and mixed well with the surrounding fluid before finally impact at the bottom of the tundish with a relatively low speed (less than $0.3 \mathrm{~m} / \mathrm{s}$ ). This is helpful to enlarge the plug volume and improve the mixing in the tundish, sequentially boosting 
opportunities to float inclusions ${ }^{[7,21]}$.

Erosion is a challenging issue for the tundish lining. It is unprocurable to measure tundish lining wear by any of the models developed in this study. It is, however, possible to associate the areas of lining wearing to the wall skin friction coefficient (nondimensional), which is directly proportional to shear stress between fluid flow and tundish wall. Figure 11 shows the mean skin friction coefficient distribution of the tundish wall under the two ladle shrouds of 80 seconds' average (considering the limited calculating time, the contour is not totally symmetric as expected, but enough to capture the main features). The maximum coefficient, presented at the bottom wall, reaches to 3.1 for the CLS and 1.0 for the TLS respectively. Thus, the tundish wall is effectively protected from being prematurely worn with respect to fluid flow under the TLS.

Tundish slag is not considered in the current investigation to determine the slag entrapment. The velocity of free surface of a liquid can work as an index to assess the state of liquid level. As shown in Figure 12(a), the mean speed of the free surface ranges from $0.01 \mathrm{~m} / \mathrm{s}$ to $0.17 \mathrm{~m} / \mathrm{s}$ and peaks at the left side of the shroud for the CLS. When the TLS is used, the maximum value decreases almost $50 \%$ and reaches to $0.09 \mathrm{~m} / \mathrm{s}$ (see Figure 12(b)) and the velocity of the free surface appears to be more even at the same time. This can be illustrated from the flow pattern in the tundish (see Figures 9 and 10). The jet of the TLS mixes with more fluid at low velocity in the tundish and the velocity of the upflow from the bottom to the free surface becomes weaker, thus a relatively quiet liquid surface is formed. These advantages of the TLS can be extended to tundish filling and ladle change operations, maintaining minimal turbulence and permitting safe submerged opening ${ }^{[7,8]}$.

The residence time distribution (RTD) characteristics and curve profiles of studied cases are 
given in Table III and Figure 13. It can be observed that when the CLS is replaced by the CLS, the plug volume is increased from $4.12 \%$ to $11.29 \%$, and the dead volume is reduced from $28.00 \%$ to $17.91 \%$ respectively. The entering jet of the TLS is at low velocity and minimises the turbulence inside the tundish, which contributes to a longer breakthrough time $(1.5 \mathrm{~s}, 2.2 \mathrm{~s}$ for the CLS and TLS respectively) and peak time (2.0 s, $7.4 \mathrm{~s}$ for the CLS and TLS respectively). Meanwhile, the swinging and distorted jet of the TLS enhances the mixing behavior inside the tundish and reduces the dead volume. For the RTD curve of the TLS, the concentration peaks at a lower height than that of the CLS and gently falls down, which confirms the improved mixing characteristics. Thus, the application of the TLS is beneficial to the flow characteristics, and thereby facilitating the inclusion flotation.

It should be pointed out that the current study only focuses on a one-third scale modeling of the two types of ladle shroud and a simplified tundish model due to challenges to apply LES model in full scale study. Although our results reflect the primary behaviors of the fluid flow and mass transfer inside the two ladle shroud and a tundish model, future work should be extended to have a better understanding of the industrial practices.

\section{Conclusions}

A comparative study of the fluid dynamics and mass transfer in a conventional ladle shroud and a trumpet ladle shroud has been carried out. The feasibility and mechanisms of the trumpet ladle shroud to enhance producing efficiency and molten steel quality have been determined with respect to fluid flow, and a summary Figure $\mathbf{1 4}$ is depicted to clarify the relationships. The following conclusions can be drawn from the results:

1. Compared with the flow pattern in the CLS, the flow velocity decreases and strong vortices 
are formed in the trumpet section of the TLS which promotes higher velocity fluctuations and turbulence dissipation rate. The outflow leaves out the ladle shroud at an average speed of 0.27 to $0.41 \mathrm{~m} / \mathrm{s}$ when the inlet velocity is $0.708 \mathrm{~m} / \mathrm{s}$ for the TLS.

2. The trumpet section of the TLS enlarges the volume of the nozzle and contributes to upward and distorted flow along the nozzle wall, which limits the transport and attachment of inclusions to the inner wall and finally eliminates the nozzle clogging.

3. The TLS changes the vorticity intensity and the location of the strongest vortex with the help of the trumpet section to alter the flow pattern inside the ladle shroud and the tundish, thereby weakening the liquid level fluctuation and the tendency of entrapping tundish slag.

4. When the flow rate is the same for the two ladle shrouds, the jet of the TLS has a lower velocity and stronger fluctuations than those of the CLS, thus having lower entering energy into the tundish. The outflow of the TLS gets twisted and is mixed with more fluid in the tundish, enhancing the turbulent energy dissipation. These two effects contribute to the reductions of the mean skin friction and free surface velocity.

5. The RTD characteristics demonstrated better mixing behaviors inside the tundish model in terms of increased plug volume and decreased dead volume, when the TLS is used. 


\section{ACKNOWLEDGEMENTS}

The authors would like to appreciate the technique support of Mr. Dongxia Li for the PIV experiments. This research is supported by National Science Foundation of China (No. 51304016 and 51474076), also funded by Research and Development Funds of State Key Laboratory of Advanced Metallurgy, No. 41603014. One of the authors, Jiangshan Zhang would like to thank the funding of Chinese Scholar Council (CSC) for his study at UOW. 


\section{NOMENCLATURE AND ACRONYMS}

\section{GREEK SYMBOLS}

$\begin{array}{ll}\phi & \text { Any function } \\ \phi^{\prime \prime} & \text { Sub-grid component } \\ \bar{\phi} & \text { Filtered function } \\ \rho & \text { Mass density }\left(\mathrm{kg} / \mathrm{m}^{3}\right) \\ \tau_{i j} & \text { SGS stress }(\mathrm{N}) \\ \tau_{k k} & \text { Isotropic part of the SGS stresses }(\mathrm{N}) \\ v_{0} & \text { Molecule viscosity }(\mathrm{Kg} /(\mathrm{m} \cdot \mathrm{s})) \\ v_{\mathrm{t}} & \text { Turbulent eddy-viscosity }(\mathrm{Kg} /(\mathrm{m} \cdot \mathrm{s})) \\ v_{\mathrm{eff}} & \text { Effective viscosity }(\mathrm{Kg} /(\mathrm{m} \cdot \mathrm{s})) \\ \Delta & \text { Mesh length }(\mathrm{m}) \\ \Delta t & \text { Time step second }(\mathrm{s}) \\ \zeta_{i j} & \text { Vorticity tensor } \\ \kappa & \text { Von Kármán constant } \\ \delta_{i j} & \text { Kronecker symbol } \\ \varepsilon & \text { Dissipation rate of kinetic energy }\left(\mathrm{m}^{2} / \mathrm{s}^{3}\right) \\ \vartheta_{i j} & \text { Stress tensor due to molecular viscosity }\end{array}$

\section{ROMAN SYMBOLS}

$G \quad$ Filter function

$u_{i} \quad$ Velocity in direction " $i$ " $(\mathrm{m} / \mathrm{s})$

$V \quad$ Volume of the computational cell $\left(\mathrm{m}^{3}\right)$

$t \quad$ Time (s)

$p \quad$ Pressure $(\mathrm{Pa})$

$S \quad$ Rate of strain tensor

$D_{\text {eff }} \quad$ Effective mass transfer coefficient $\left(\mathrm{m}^{2} / \mathrm{s}\right)$

$\mathrm{Sc}_{\mathrm{t}} \quad$ Schmidt number

$D_{i j} \quad$ Deformation tensor

$L_{s} \quad$ Mixing length for sub-grid scales (m)

$\bar{g}_{i j} \quad$ Velocity gradient

$C_{w}, C_{k k}$ and $C_{v} \quad$ Constants in turbulence model

$C \quad$ Concentration of the tracer $(\mathrm{mol} / \mathrm{L})$ 


\section{REFERENCES}

1. Y. Sahai and T. Emi: Tundish Technology for Clean Steel Production, World Scientific Publishing Company, Hackensack, NJ, 2008, pp. 9-14.

2. S. Yang, L. Zhang, J. Li and K. Peaslee: ISIJ Int., 2009, vol. 49, pp. 1551-60.

3. A. Kumar, D. Mazumdar and S. C. Koria: ISIJ Int., 2008, vol. 48, pp. 38-47.

4. C. Chen, L. Jonsson, A. Tilliander, G. Cheng and P. Jönsson: Metall. Mater. Trans. B, 2015, vol. 46, pp. 169-90.

5. R. Schwarze, D. Haubold and C. Kratzsch: Ironmak. Steelmak., 2015, vol. 42, pp. 148-53.

6. K. Morales-Higa, R. Guthrie, M. Isac and R. Morales: Metall. Mater. Trans. B, 2013, vol. 44, pp. 63-79.

7. M. Nadif, J. Lehmann, M. Burty and J.-F. Domgin: Revue de Métallurgie, 2007, vol. 104, pp. 493-500.

8. B. Thomas and H. Bai: 18rd Process Technol. Steelmak. Conf. Proc., Warrendale, I\&S Society PA, March 25-28, 2001, pp 895-912.

9. L. Zhang and B. Thomas: ISIJ Int., 2003, vol. 43, pp. 271-91.

10. G. Solorio-Diaz, R. Morales, J. Palafox-Ramos and A. Ramos-Banderas: ISIJ Int., 2005, vol. 45, pp. 1129-37.

11. J. Zhang, S. Yang, J. Li, W. Yang, Y. Wang and X. Guo: ISIJ Int., 2015, vol. 55, pp.1684-92.

12. K. Chattopadhyay, M. Hasan, M. Isac and R. L. Guthrie: Metall. Mater. Trans. B, 2010, vol. 41, pp. 225-33.

13. B. Becker and N. Prabhu: 9th Process Technol. Steelmak. Conf. Proc., Washington, DC 
1991, April 14-17, pp. 489-93.

14. G. Wen, Y. Huang and P. Tang: J. Chongqing Univ., 2011, vol. 34, pp. 69-74.

15. J. Wu and H. Wang: Chinese Patent, No. CN 202701369 U.

16. K. Chattopadhyay: Modelliing of Transport Phenomena for Improved Steel Quality in a Delta Shaped Four Strand Tundish, Ph.D Thesis, McGill University, 2011.

17. S. Ghosal: AIAA J., 1999, vol. 37, pp. 425-33.

18. G. Solorio-Diaz, R. Morales, J. Palafax-Ramos, L. Garcia-Demedices and A. Ramos-Banderas: ISIJ Int., 2004, vol. 44, pp. 1024-32.

19. Q. Yuan, S. Vanka, B. Thomas and S. Sivaramakrishnan: Metall. Mater. Trans. B, 2004, vol. 35 , pp. $967-82$.

20. Z. Qian and Y. Wu: ISIJ Int., 2004, vol. 44, pp. 100-07.

21. W. W. Kim: A New Dynamic Subgrid-Scale Model for Large-Eddy Simulation of Turbulent Flows, Ph.D thesis, Georgia Institute of Technology, Georgia, USA, 1996.

22. ANSYS Inc. FLUENT6.3-Manual, ANSYS Inc., Lebanon, NH,. 2007.

23. Y. Sahai and T. Emi: ISIJ Int., 1996, vol. 36, pp. 667-72.

24. U. Piomelli and E. Balaras: Annu. Rev. Fluid Mech., 2002, vol. 34, pp. 349-74.

25. M. Lesieur and O. Metais: Annu. Rev. Fluid Mech., 1996, vol. 28, pp. 45-82.

26. F. Wilson, M. Heesom, A. Nicholson and A. Hills: Ironmak. Steelmak., 1987, vol. 14, pp. 296-309. 


\section{Table Captions:}

Table I. Documented benefits of the TLS in available literature

Table II. Tundish parameters and fluid properties

Table III. RTD characteristics of studied cases

\section{Figure Captions:}

Fig. 1- (a) Schemes of studied ladle shrouds. (b) Mesh of the model.

Fig. 2 - The scheme of physical experiment apparatus

Fig. 3- Velocity vectors under the trumpet-shaped ladle shroud: (a), (c) and (e): LES results; (b), (d) and (f): PIV results.

Fig. 4- Instantaneous velocity histories at three points of the two LS outlets: (a) CLS; (b) TLS.

Fig. 5- Typical velocity profiles at the ladle shroud centerlines. (a) CLS. (b) TLS.

Fig. 6-Typical velocity vector profiles at the transversal XZ plane. (a) CLS. (b) TLS, with upward flow vectors in areas (1) and (2), curved shape flow vectors in area (3). (The density of vectors is reduced to clearly show the vectors in the above profiles)

Fig. 7- Typical Strain Rate profiles at the ladle shroud centerlines. (a) CLS. (b) TLS.

Fig. 8 - Typical evolution tracks of vortices on XZ plane. (a) CLS. (b) TLS. (Vortices are circled)

Fig. 9- Typical flow patterns under the CLS on XZ plane, (a) straight downward flow, (b) swinging towards the right, (c) swinging towards the right, (d) twisted flow.

Fig. 10 - Typical flow patterns under the TLS on XZ plane, (a) straight downward flow, (b) swinging towards the right, (c) swinging towards the right, (d) twisted flow.

Fig. 11- Mean skin friction coefficient profiles of bottom tundish walls under the two shrouds. (a) CLS. (b) TLS.

Fig. 12- Mean velocity of the free surface with the two ladle shrouds. (a) CLS. (b) TLS.

Fig. 13 - RTD curves of the studied cases

Fig. 14- Possible contributing flow and mass transfer mechanisms for benefits of the TLS 
Table I. Documented benefits of the TLS in available literature

\begin{tabular}{|c|c|}
\hline Authors/year & Main benefits of the TLS \\
\hline B. Becker et al./1991 ${ }^{[13]}$ & $\begin{array}{l}\text { - Decreased clogging rate and improved producing efficiency } \\
\text { - Less folding in cold rolling process at No. } 2 \text { BOF/CC of Inland } \\
\text { Steel, combined with a steel sheet cover at the end of the TLS }\end{array}$ \\
\hline B. G. Thomas et al/2001 ${ }^{[8]}$ & - $\quad$ Maintaining minimal turbulence during tundish filling \\
\hline L. Zhang et al./2003 ${ }^{[9]}$ & - Lower T.O contents were observed inside the tundish for 300 heats \\
\hline G. Solorio-Díaz et al./2004 ${ }^{[18]}$ & $\begin{array}{l}\text { - The tip of the shroud has bell shape to reinforce the braking effect } \\
\text { on the fluid which flows into the tundish. }\end{array}$ \\
\hline M. Nadif et al./2007 $7^{[7]}$ & $\begin{array}{l}\text { - Permitting safe submerged opening with bell-shaped } \\
\text { - Preventing the generation and entrapment in products of emulsified } \\
\text { steel-slag mix }\end{array}$ \\
\hline K.Chattopadhyay/2011 $1^{[16]}$ & $\begin{array}{l}\text { - } \quad \text { Eliminating flow backs during a submerged ladle change operation. } \\
\text { - Accommodating for hot gas pockets. }\end{array}$ \\
\hline G. Wen et al./2011 ${ }^{[14]}$ & $\begin{array}{l}\text { - Decreasing shortcut flow volume; elongating residence time and } \\
\text { improving mixing in physical experiments }\end{array}$ \\
\hline J.Wu et al./2013 $3^{[15]}$ & $\begin{array}{l}\text { - Weakening the back flow inside the ladle shroud during the tundish } \\
\text { filling }\end{array}$ \\
\hline
\end{tabular}


Table II. Tundish parameters and fluid properties

\begin{tabular}{ll}
\hline Parameters & Values \\
\hline Length of the domain, $\mathrm{mm}$ & 500.0 \\
Height of the domain, $\mathrm{mm}$ & 170.0 \\
Width of the domain, $\mathrm{mm}$ & 250.0 \\
Submergence depth of shroud, mm & 36.5 \\
Inlet diameter of shroud d1, mm & 30.0 \\
Outlet diameter of shroud d2, mm & 50.0 \\
Water density, $\mathrm{kg} / \mathrm{m}^{3}$ & 1000 \\
Water viscosity, $\mathrm{kg} /(\mathrm{m} \cdot \mathrm{s})$ & 0.001 \\
Flow volume rate, $\mathrm{L} / \mathrm{min}$ & 30 \\
Whole Length of the shrouds $(\mathrm{L}), \mathrm{mm}$ & 412.5 \\
Height of the trumpet section $(\mathrm{h}), \mathrm{mm}$ & 200.0 \\
\hline
\end{tabular}


Table III. RTD characteristics of studied cases

\begin{tabular}{cccccc}
\hline & $\begin{array}{c}\text { Plug volume } \\
(\%)\end{array}$ & $\begin{array}{c}\text { Dead } \\
\text { volume (\%) }\end{array}$ & $\begin{array}{c}\text { Mixed } \\
\text { volume (\%) }\end{array}$ & $\begin{array}{c}\text { Breakthrough } \\
\text { time (s) }\end{array}$ & $\begin{array}{c}\text { Peak time } \\
(\mathrm{s})\end{array}$ \\
\hline CLS & 4.12 & 28.00 & 67.88 & 1.5 & 2.0 \\
TLS & 11.29 & 17.91 & 70.80 & 2.2 & 7.4 \\
\hline
\end{tabular}




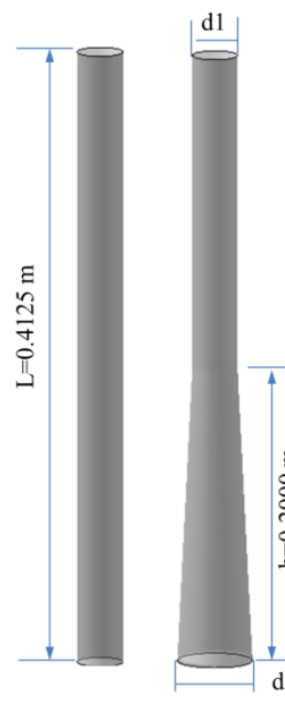

(a)

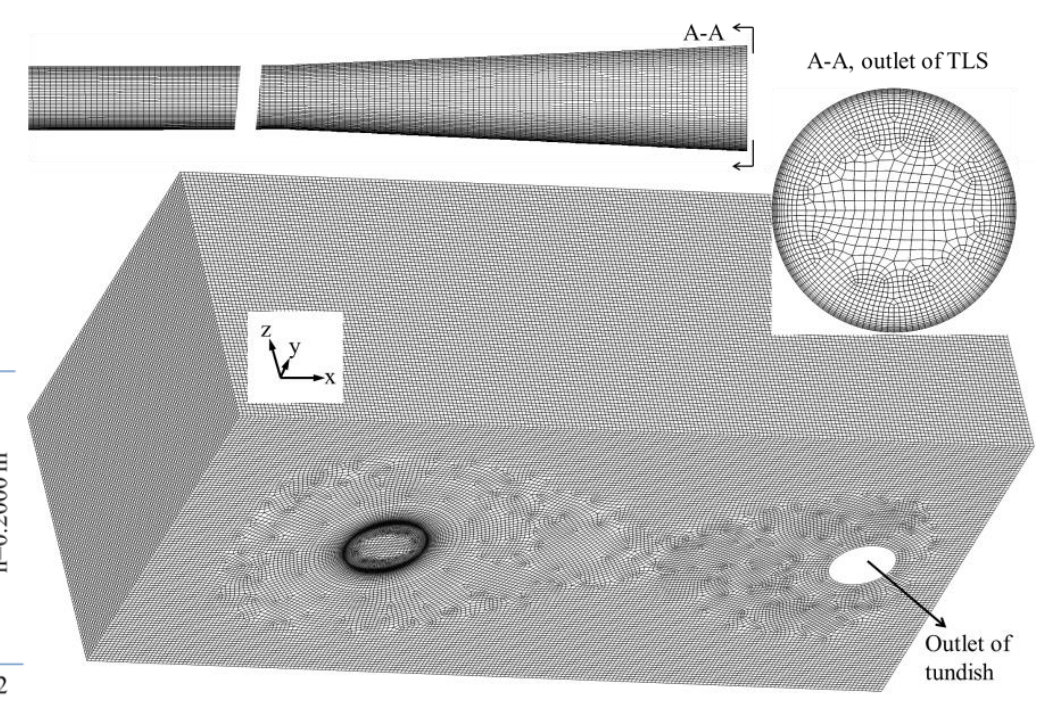

(b)

Fig. 1- (a) Schemes of studied ladle shrouds. (b) Mesh of the model. 


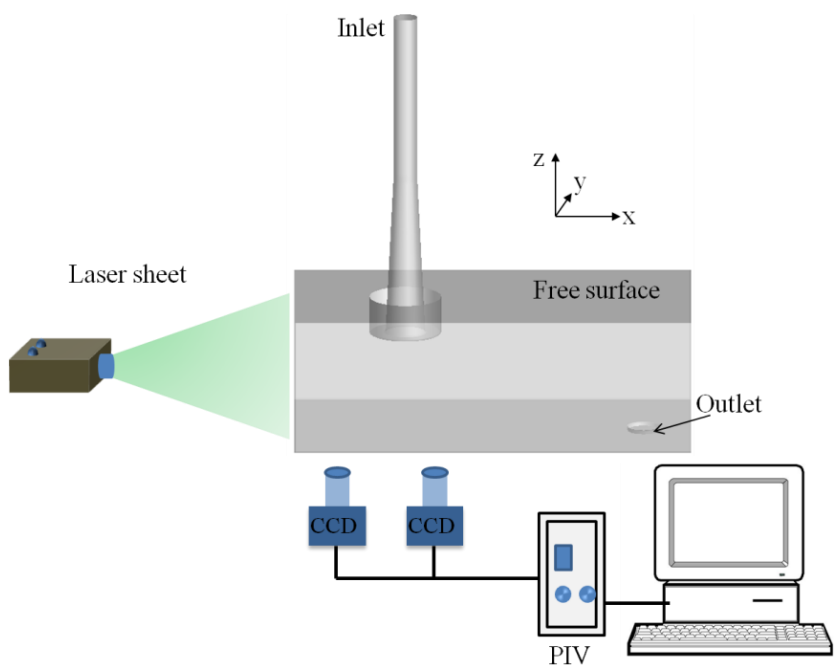

Fig. 2- The scheme of physical experiment apparatus 


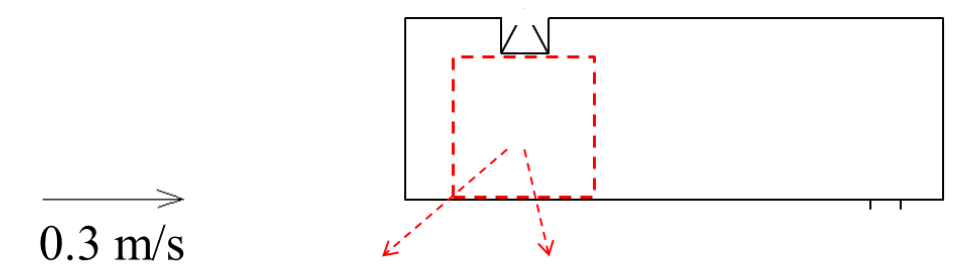

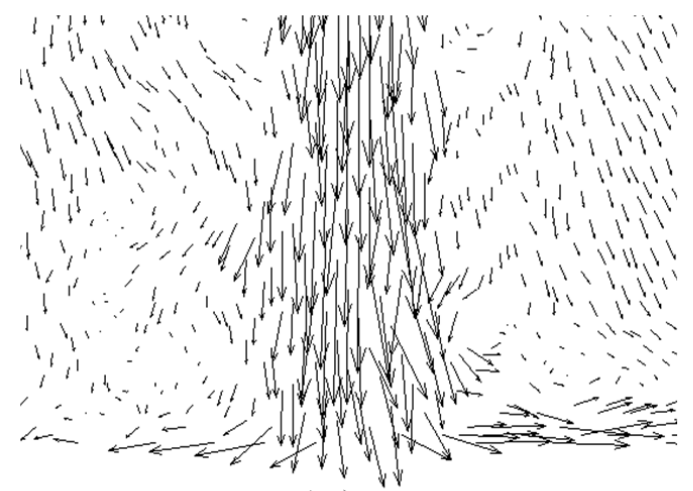

(a)

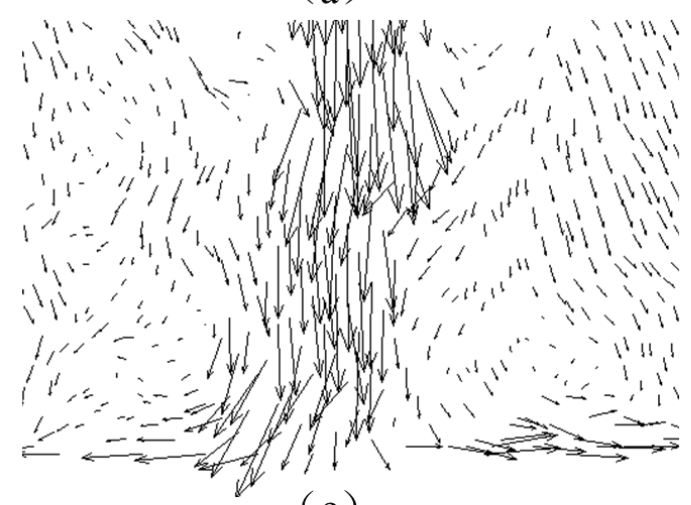

(c)

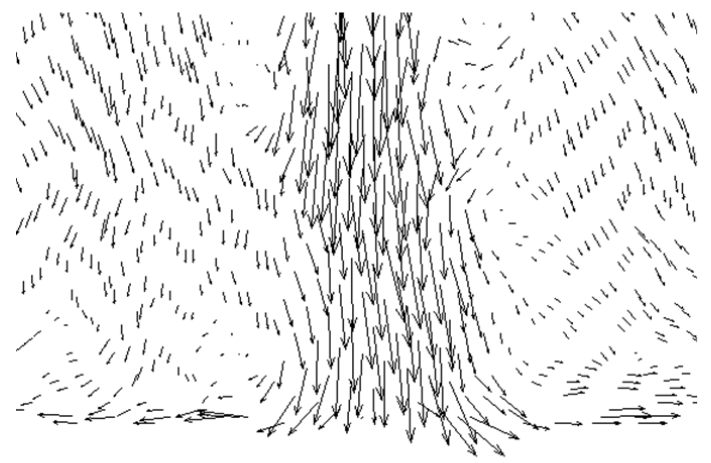

(e)

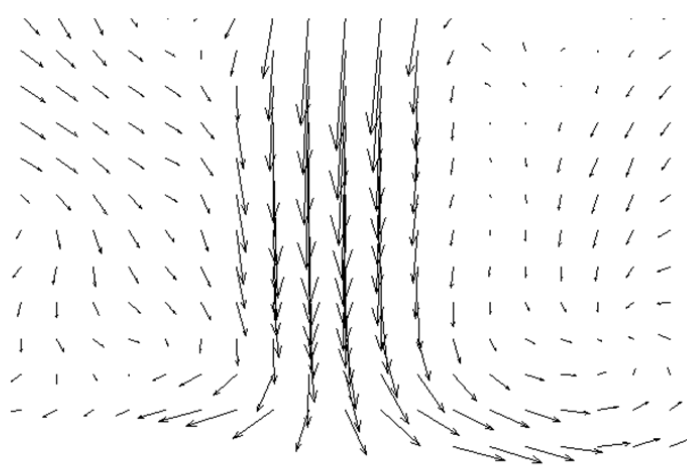

(b)

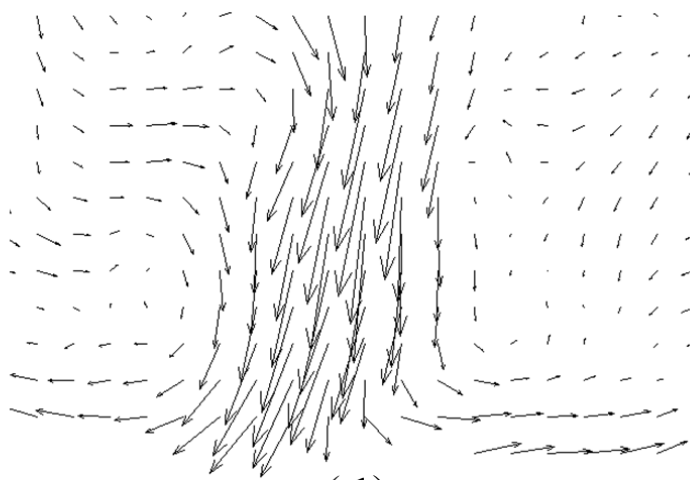

(d)

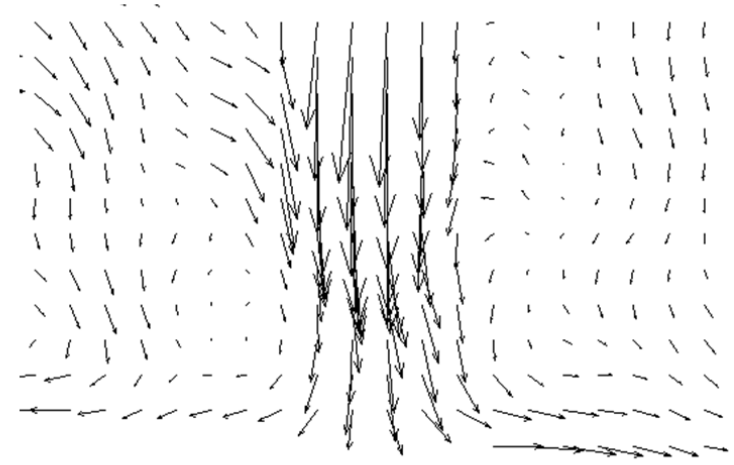

(f)

Fig. 3 - Velocity vectors under the trumpet-shaped ladle shroud: (a), (c) and (e): LES results; (b), (d) and (f): PIV results. 


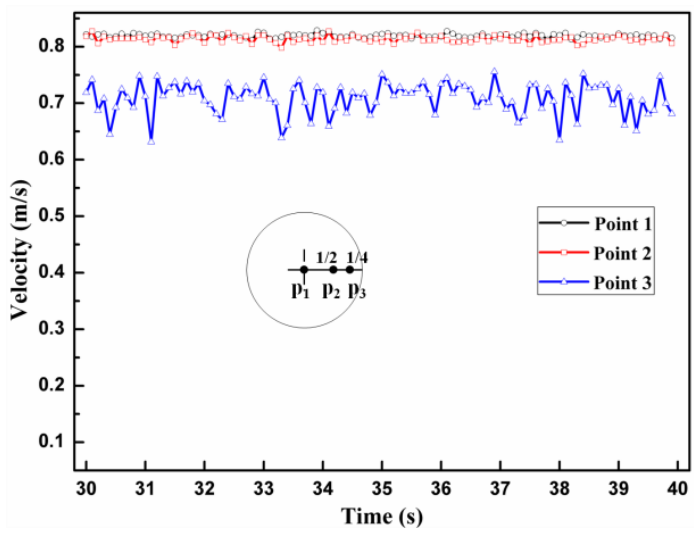

(a)

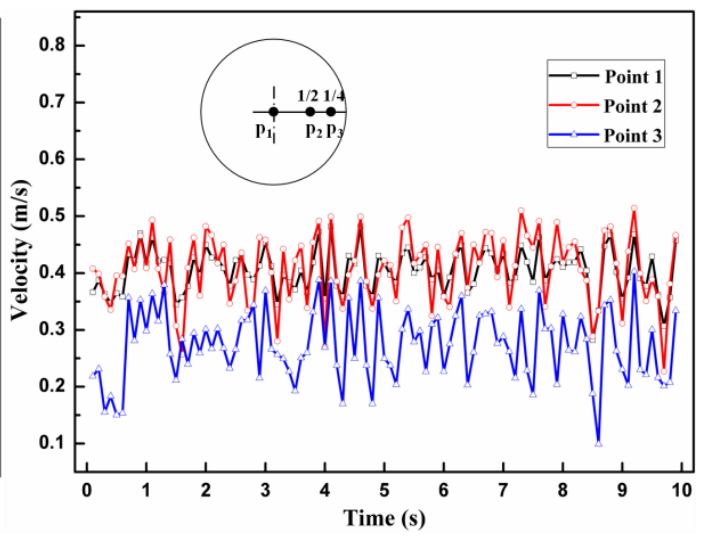

(b)

Fig. 4- Instantaneous velocity histories at three points of the two LS outlets: (a) CLS; (b) TLS. 


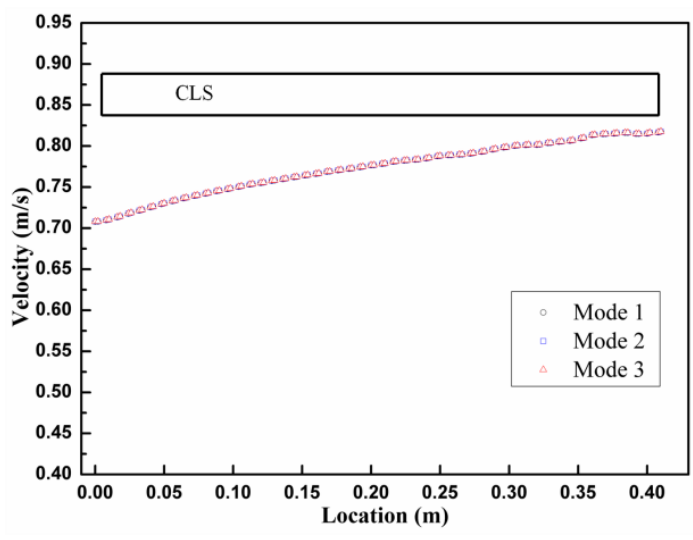

(a)

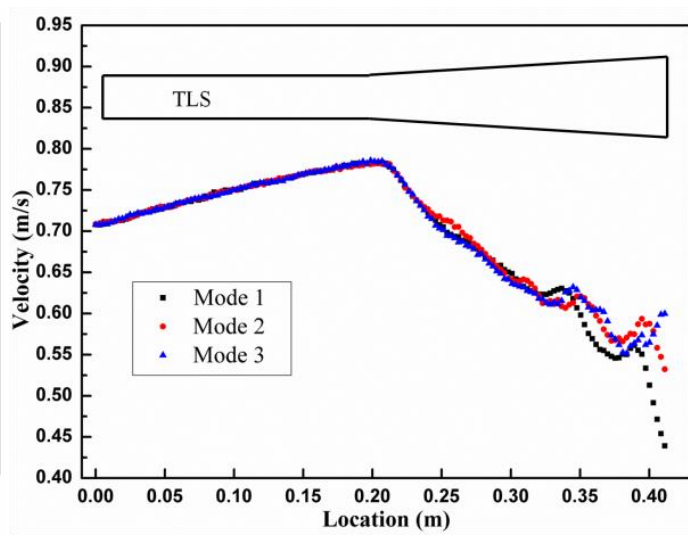

(b)

Fig. 5- Typical velocity profiles at the ladle shroud centerlines. (a) CLS. (b) TLS. 


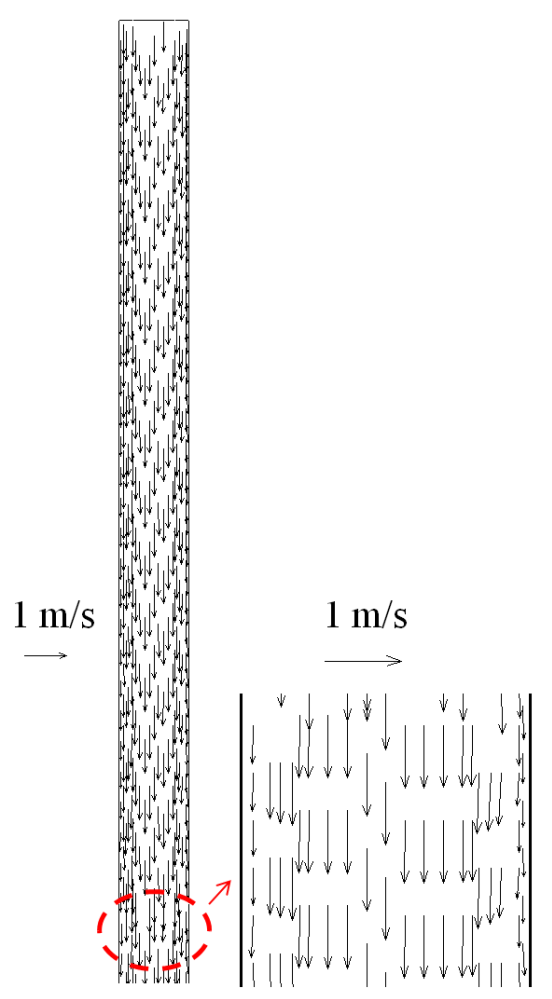

(a)

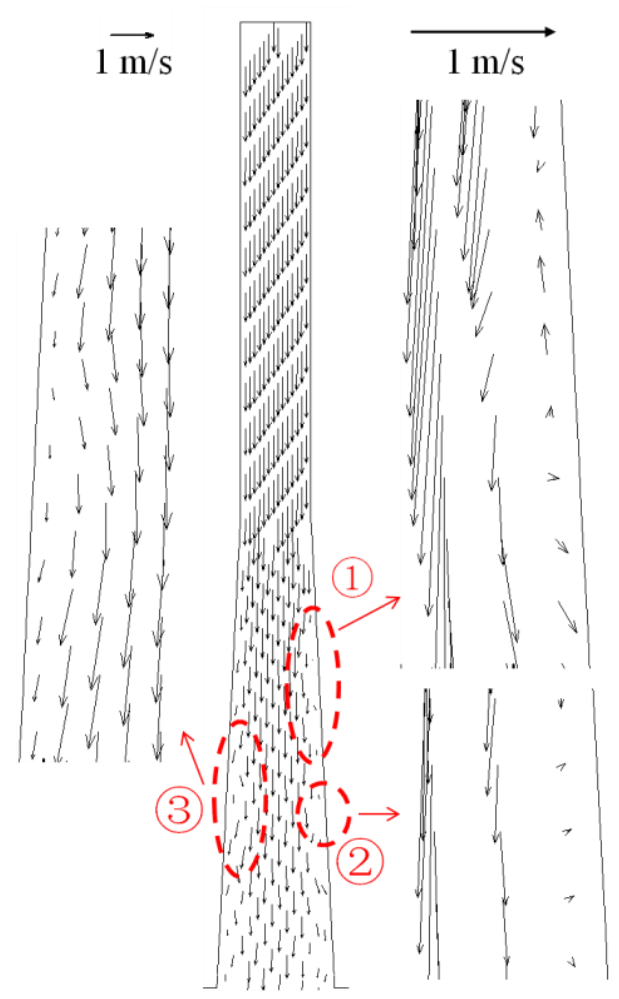

(b)

Fig. 6- Typical velocity vector profiles at the transversal XZ plane. (a) CLS. (b) TLS, with upward flow vectors in areas (1) and (2), curved shape flow vectors in area (3). (The density of vectors is reduced to clearly show the vectors in the above profiles) 


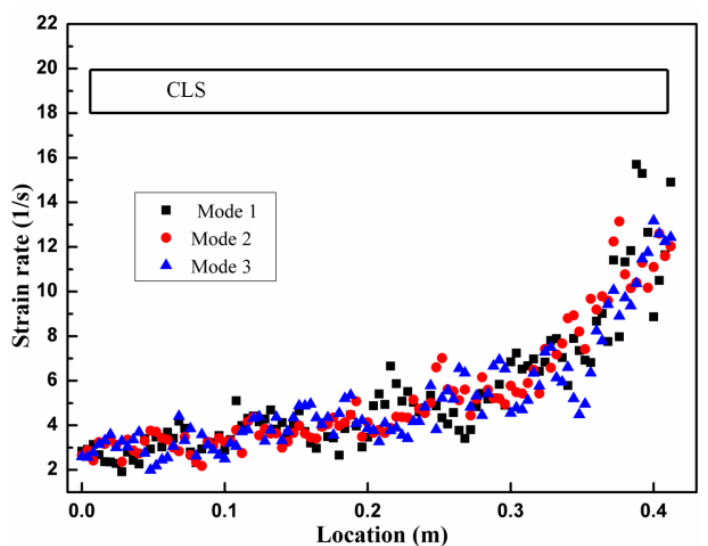

(a)

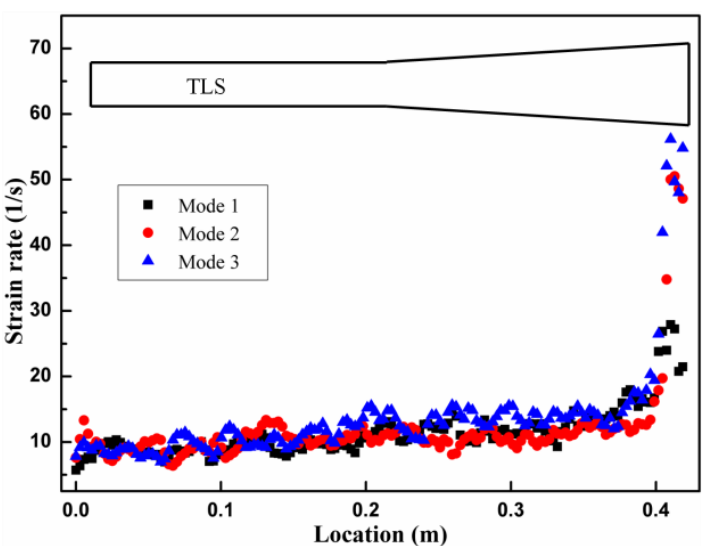

(b)

Fig. 7- Typical Strain Rate profiles at the ladle shroud centerlines. (a) CLS. (b) TLS. 

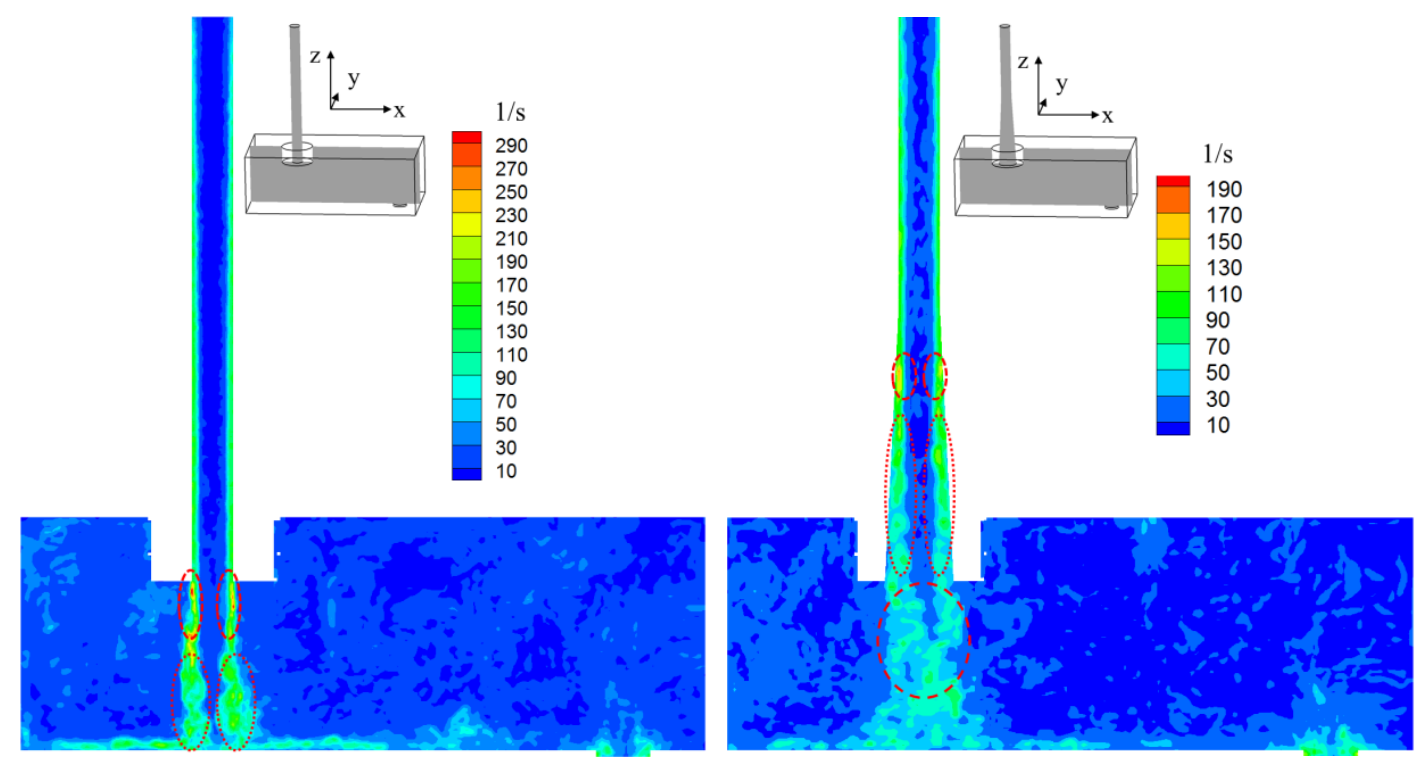

Fig. 8- Typical evolution tracks of vortices on XZ plane. (a) CLS. (b) TLS. (Vortices are circled) 


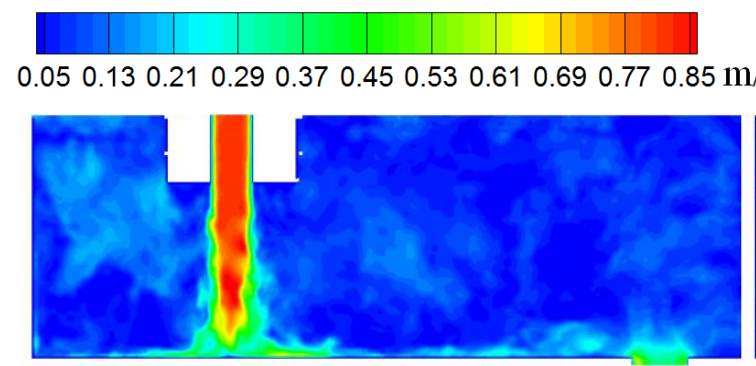

(a)

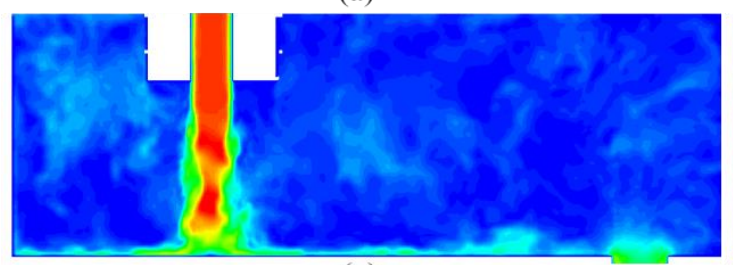

(c)

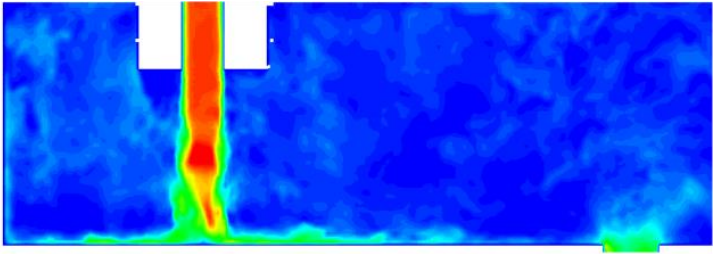

(b)

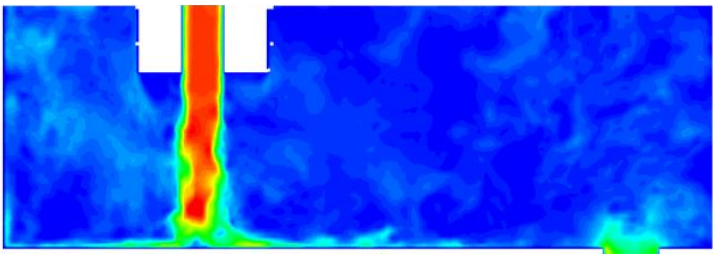

(d)

Fig. 9- Typical flow patterns under the CLS on XZ plane, (a) straight downward flow, (b) swinging towards the right, (c) swinging towards the right, (d) twisted flow. 


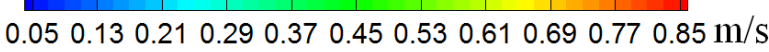

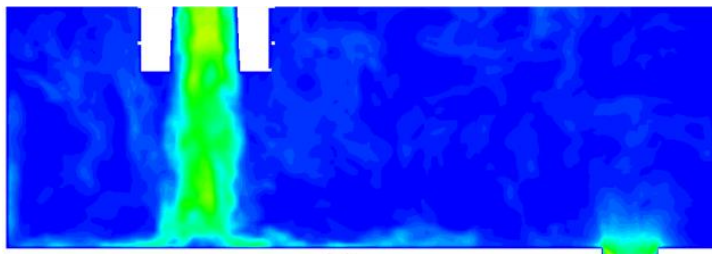

(a)

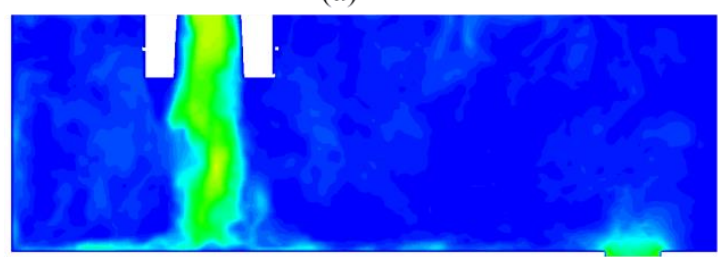

(c)

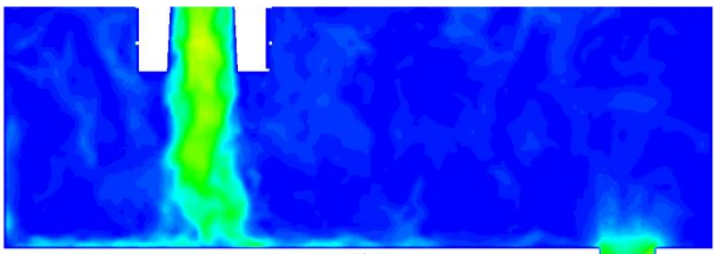

(b)

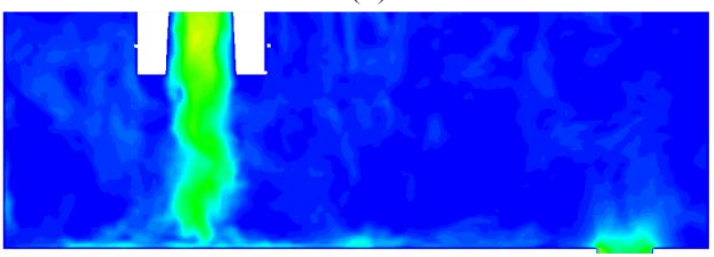

(d)

Fig. 10 - Typical flow patterns under the TLS on XZ plane, (a) straight downward flow, (b) swinging towards the right, (c) swinging towards the right, (d) twisted flow. 


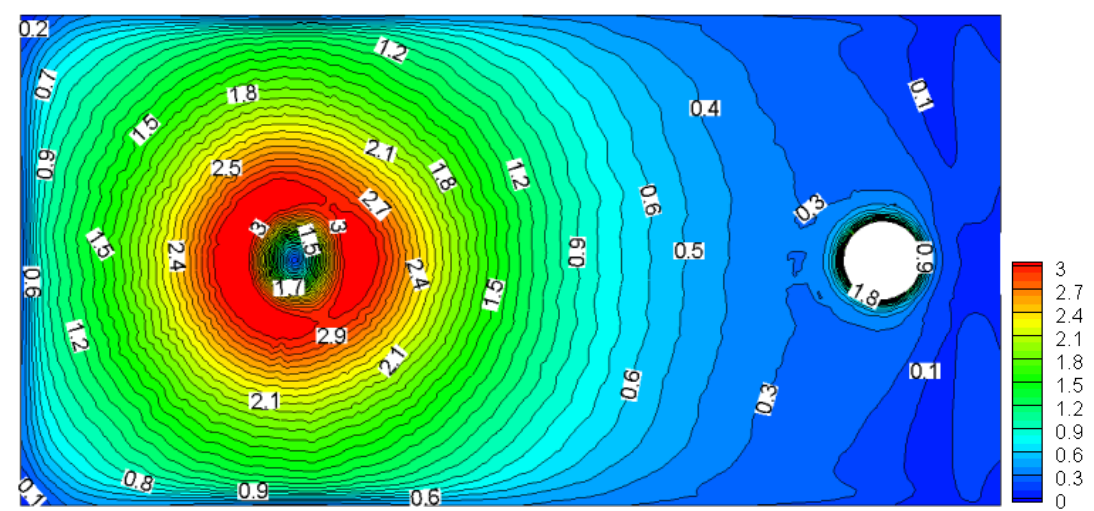

(a)

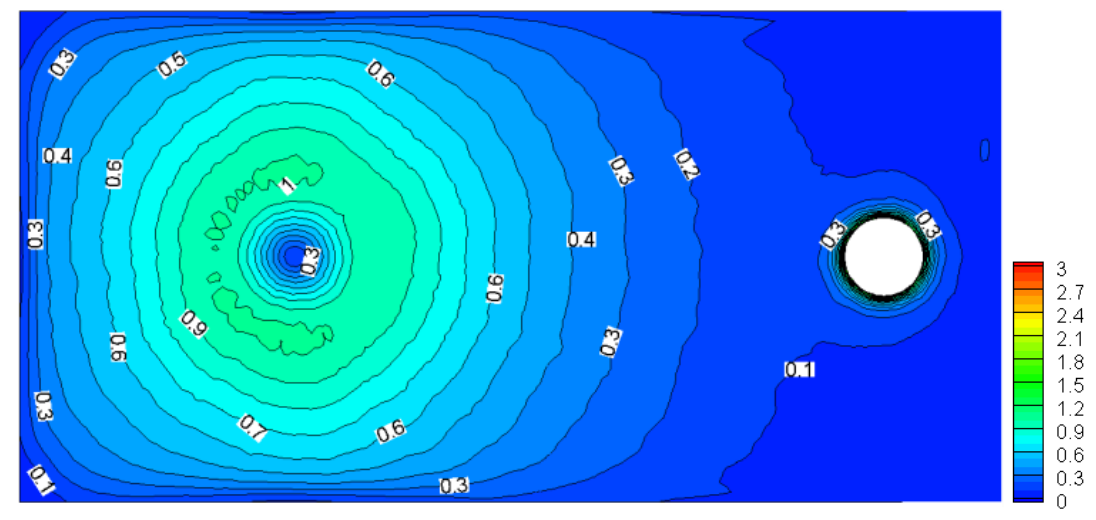

(b)

Fig. 11- Mean skin friction coefficient profiles of bottom tundish walls under the two shrouds. (a) CLS. (b) TLS. 


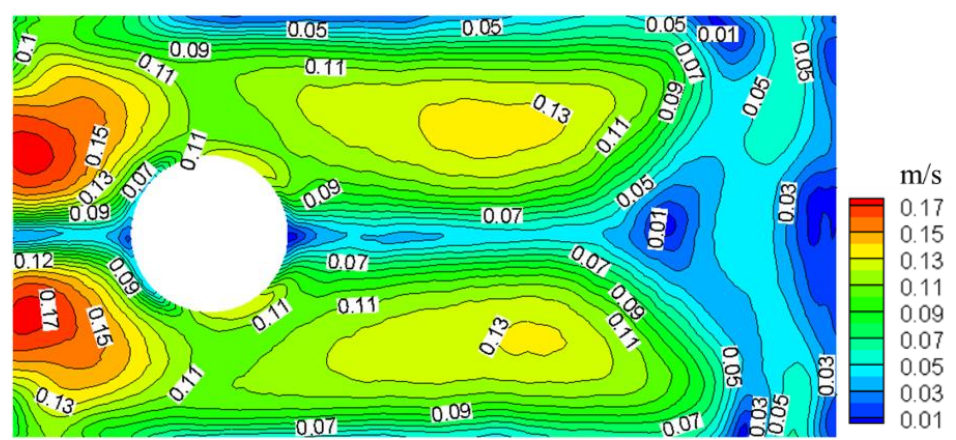

(a)

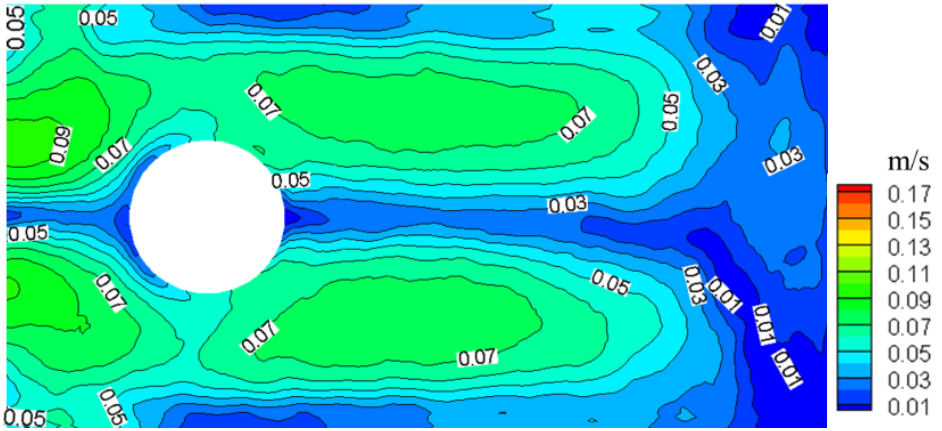

(b)

Fig. 12- Mean velocity of the free surface with the two ladle shrouds. (a) CLS. (b) TLS. 


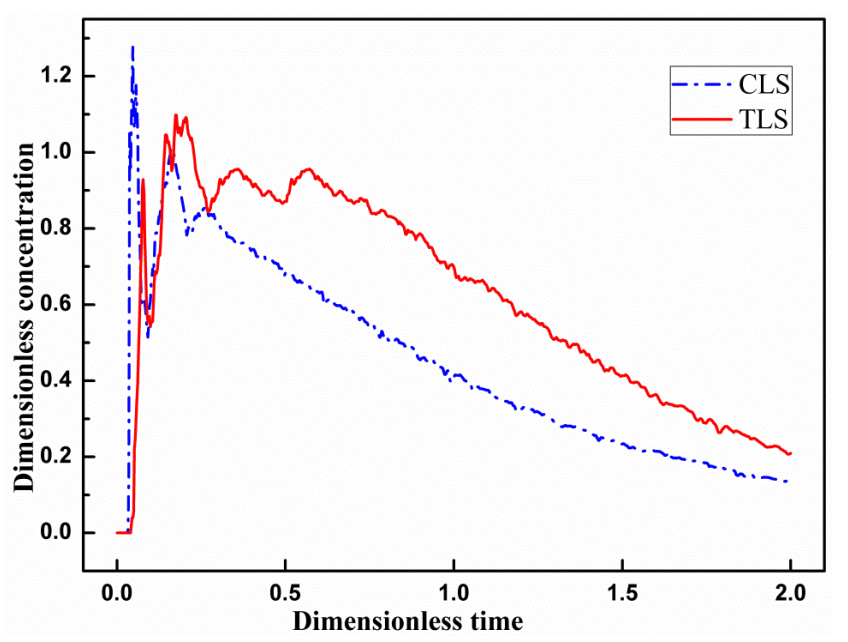

Fig. 13 - RTD curves of the studied cases 


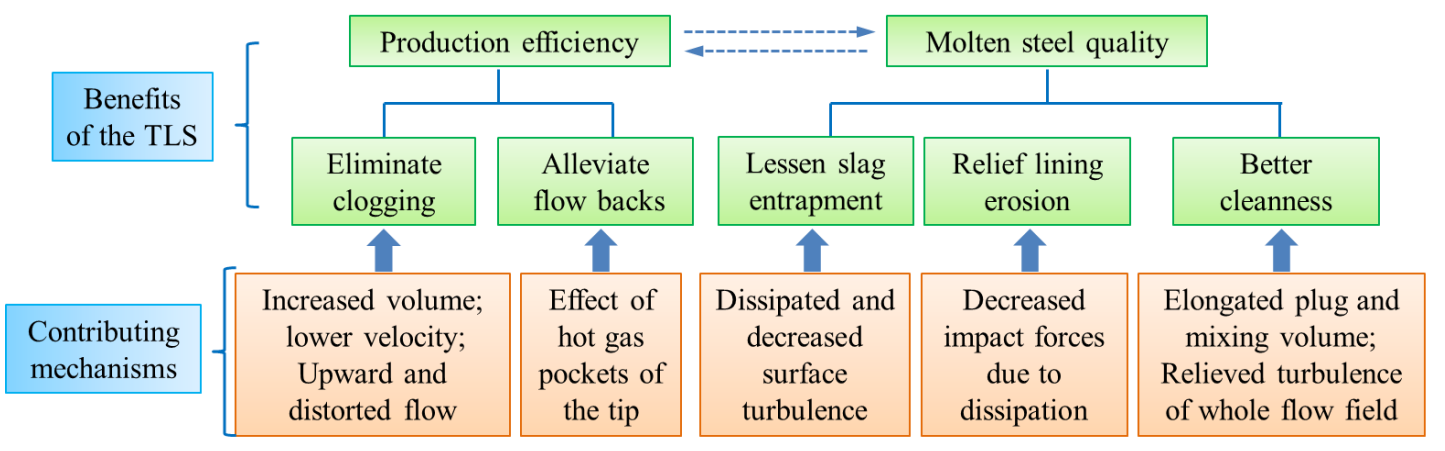

Fig. 14- Possible contributing mechanisms of flow and mass transfer for benefits of the TLS 